\title{
Position-Dependent Diffusion Dynamics of Entangled Polymer Melts Nanoconfined by Parallel Immiscible Polymer Films
}

\author{
Kyoung-Il Jo ${ }^{a, b}$, Younghoon Oh', Tae-Ho Kim ${ }^{d}$, Joona Bang ${ }^{b}$, Guangcui Yuan ${ }^{e}$, \\ Sushil K. Satija ${ }^{e}$, Bong June Sung ${ }^{c}$, Jaseung Koo ${ }^{d}$ *
}

${ }^{a}$ Neutron Science Center, Korea Atomic Energy Research Institute (KAERI), Daejeon 34057 , Korea

${ }^{b}$ Department of Chemical and Biological Engineering, Korea University, Seoul 02841, Korea 'Department of Chemistry and Research Institute for Basic Science, Sogang University, Seoul 04107, Korea

dDepartment of Organic Materials Engineering, Chungnam National University, Daejeon 34134, Korea

eNIST Center for Neutron Research, National Institute of Standards and Technology, Gaithersburg, MD 20899, United States

Corresponding author: jkoo@cnu.ac.kr 


\section{EXPERIMENTAL SECTION}

Materials We purchased poly(methyl methacrylate) (PMMA) $\left(\mathrm{M}_{\mathrm{w}}=92,000\right.$ $\left.\mathrm{g} / \mathrm{mol}, M_{\mathrm{w}} / M_{\mathrm{n}}=1.06\right)$, deuterated PMMA $\left(d \mathrm{PMMA} ; M_{\mathrm{w}}=106,000 \mathrm{~g} / \mathrm{mol}, M_{\mathrm{w}} / M_{\mathrm{n}}=1.60\right)$ and polystyrene (PS) $\left(M_{\mathrm{w}}=7,100,000 \mathrm{~g} / \mathrm{mol}, M_{\mathrm{w}} / M_{\mathrm{n}}=1.15\right)$ from Polymer Source Inc. (Dorcal, Quebec, Canada). All solvents were purchased from Sigma Aldrich (St. Louis, Missouri, USA). Silicon (Si) substrate preparation. Polished 3" and 4" Silicon (Si) substrates (100) wafers were purchased from Shin-Etsu (Tokyo, Japan). Their surfaces were rinsed with deionized(DI) water. After drying, they were exposed to UV/ozone (UV/Ozone ProCleaner, BioForce Nanoscience, Ames, IA) to remove remaining organic contamination. Next, the substrates were immersed in about $20 \mathrm{ml}$ of diluted hydrofluoric acid (HF) solution $\left(\mathrm{H}_{2} \mathrm{O}\right.$ : $\mathrm{HF}=10: 1)$ to etch the oxide layer. The etched wafers were then thoroughly cleaned with DI water, followed by blowing with a $\mathrm{N}_{2}$ gas stream.

Polymer multilayers for diffusion dynamics study The PS and (d)PMMA were dissolved in 1-chloropentane and toluene, respectively, at the various concentrations to obtain the desired thicknesses. The solutions were filtered through PTFE membranes ( 1 and $0.5 \mu \mathrm{m}$, Milipore, Billerica, MA, USA). First, the UV/ozone was exposed to the wafer surfaces for $90 \mathrm{~min}$ in advance. The $d$ PMMA solution were spin-coated on UV/ozone-treated hydrophilic 4" Si wafer. In addition, the PS solution at the concentration of $2.5 \mathrm{mg} / \mathrm{ml}$ was spun-cast on the 3 " Si slabs ( $5 \mathrm{~mm}$ thick) at 2,500 rpm. The multilayers were dried in a vacuum oven at room temperature overnight to remove any excess solvent trapped in the films. The thicknesses of films were checked by an Ellipsometer (SE MG-1000 UV, Nano-View Co., Ltd., Korea) in advance to compare with the neutron reflectivity results. The $d$ PMMA thin films were carefully floated on DI water surface. These were then transferred onto the PS thin film on the HF-etched Si 
surfaces (denoted as a $d \mathrm{PMMA} / \mathrm{PS} / \mathrm{Si}$ ). These bilayer samples were dried in a vacuum at room temperature for $24 \mathrm{~h}$. For the bilayer of PS/PMMA, the PMMA solution were spin-coated on UV/ozone-treated substrate in advance. Then, the PS solution in 1-chloropentane $(2.5 \mathrm{mg} / \mathrm{ml})$ was directly spin-coated on the prepared PMMA surface. Direct spinning is possible because 1-chloropentane is a good solvent for the PS but a poor solvent for the PMMA. ${ }^{1}$ Similarly, the bilayer of PS/PMMA was floated on DI-water surface. Subsequently, the prepared $d \mathrm{PMMA} / \mathrm{PS} / \mathrm{Si}$ sample was juxtaposed to the PMMA/PS thin film at the air-water interface, followed by transferring in horizontal contact, resulting in a multilayer of PS/PMMA/dPMMA/PS/Si. The samples were kept in a vacuum desiccator.

Construction of Simulation Systems We perform a series of coarse-grained simulations with a general polymer model that were employed in previous studies. ${ }^{2-6}$ A polymer chain is modeled with beads that are connected via harmonic bonding potential $U_{b}=K_{b}\left(r-r_{0}\right)^{2}$, where $K_{b}=1000 \varepsilon / \sigma^{2}$ and $r_{0}=1 \sigma$. Here, $\mathrm{r}$ and $\mathrm{r}_{0}$ denotes the length between bonded monomers and desired bond length. $\sigma$ and $\varepsilon$ denote the unit of length and energy in our simulations. The degree of polymerization of the polymer chains in our simulations is fixed at 128. Since the entanglement length of the polymer model is known to $82^{7}$, polymer chains in our simulations may entangle each other to some extent. The non-bonding interactions between monomers are described via a truncated and shifted Lennard-Jones potential $U_{\alpha \beta}(r)=4 \varepsilon_{\alpha \beta}$ $\left[\left(\frac{\sigma}{r}\right)^{12}-\left(\frac{\sigma}{r}\right)^{6}\right]-U_{c}\left(r<r_{c}\right)$ and $U_{\alpha \beta}(r)=0\left(r>r_{c}\right)$ and $U_{\alpha \beta}(r)=0\left(r>r_{c}\right)$. Here, the cut-off distance $r_{c}$ is $r_{c}=2.5 \sigma . \alpha$ and $\beta$ denote the types of monomers, and $U_{c}=4 \varepsilon_{\alpha \beta}$ $\left[\left(\frac{\sigma}{r_{c}}\right)^{12}-\left(\frac{\sigma}{r_{c}}\right)^{6}\right]$. We model PS as type A polymers (upper and lower layers, Figure S1, blue) and PMMA as type B polymer (center layers, Figure S1, red). To make type A and B polymers 
interact repulsively, the interaction parameters are determined as $\varepsilon_{A A}=1, \varepsilon_{B B}=1$, and $\varepsilon_{A B}$ $=0.5$. We introduce 64 type $\mathrm{A}$ and 128 type B polymer chains to build simulation systems. Two potential walls at the upper and lower bounds of the simulation systems are applied in order to confine the polymer film. The walls and type A polymers interact via purely repulsive Weeks-Chandler-Andersen potential. ${ }^{8}$ We apply periodic boundary condition in $\mathrm{x}$ and $\mathrm{y}$ directions of the simulation box. The dimensions of the simulation box are fixed to be $20 \sigma$ in $\mathrm{x}, \mathrm{y}$ direction and $76 \sigma$ in $\mathrm{z}$ direction. The number density of monomers is 0.8 .

Molecular dynamics Simulations. We propagate our simulation systems with molecular dynamics (MD) simulations by using LAMMPS simulation package. ${ }^{9}$ We equilibrate our systems following two steps. First, we carry out MD simulations with a relatively high temperature of $T=2$ until the mean-square displacement of the chain reaches the radius of gyration of bulk polymer chains $\left(R_{g}^{\text {bulk }}=5.8\right)$. Then, we decrease the temperature down to $T=1$ and perform additional MD simulations until the potential energy of the systems converges. Velocity Verlet algorithm with a timestep of 0.01 is employed for all simulations. Temperature is fixed at 1 for the production simulations by applying Nosé-Hoover thermostat, which is higher than $T_{g}=0.43 .{ }^{10}$ We obtained 3 independent trajectories from different initial configurations.

Neutron reflectivity (NR). Specular NR technique was used to measure the interfacial structures of PS/PMMA/dPMMA/PS multilayers during annealing. The measurements were conducted by using an NG7 reflectometer at the Cold Neutron Facility of the National Institute of Standards and Technology (NIST) (Gaithersburg, MD, USA) with a monochromatic beam. The wavelength $(\lambda)$ is $4.76 \AA$ and $\Delta \lambda / \lambda$ is $\sim 0.025$. The NR data were corrected as a function of momentum transfer, $q_{\mathrm{z}}(\sim 4 \pi / \lambda \sin \theta)$, where $\theta$ and $\lambda$ are the grazing angle and wavelength of the incident neutron beam, respectively. The width of the vertical slits was gradually opened as 
a function of $q_{\mathrm{z}}$, in order to keep the resolution constant, $\Delta q_{\mathrm{z}} / q_{\mathrm{z}} \approx 0.03$. The horizontal slit width was fixed as $30 \mathrm{~mm}$. We also utilized a sample environment equipment, that is, speciallydesigned heating vacuum chamber with sapphire windows for neutron beam. in-situ NR measurements were then performed during annealing at $145^{\circ} \mathrm{C}$ and in the vacuum at $10^{-6}$ torr or less. The foot print correction and background reduction were proceeded for the obtained reflectivity profiles. The NR data were fitted to reflectivity profiles calculated from the onedimensional Schrödinger's equation of potential by the scattering length density (SLD) profile model. ${ }^{11}$ These computational profiles were computed by using a Levenberg-Marquardt nonlinear least-squares method ${ }^{12,13}$ by adjusting the thickness, SLD, and interfacial width of the unknown layers with a least-squares statistic $\left(\chi^{2}\right) . R_{\mathrm{g}}$ of the $d$ PMMA $\left(M_{\mathrm{w}}=106 \mathrm{~kg} / \mathrm{mol}\right)$ was calculated to be $8.4 \mathrm{~nm}$ according to the following equation. ${ }^{14}$

$$
\left[R_{g}^{2}\right]=\frac{b^{2} N}{6}=\frac{\left[R^{2}\right]}{6}=\frac{b^{2} M_{w} / M_{0}}{6}
$$

where $b$ is monomer length of the PMMA $(b=0.66 \mathrm{~nm})$ and $M_{0}$ is the molar mass of a monomer unit $(108.12 \mathrm{~g} / \mathrm{mol})$.

Bulk diffusion coefficient Our diffusion results were compared to the bulk diffusion coefficient of PMMA reported by Kunz and stamm, that is, $D_{\text {bulk }}=0.215 \times 10^{18} \mathrm{~cm}^{2} \mathrm{~s}^{-1}$ at 130 ${ }^{\circ} \mathrm{C} .{ }^{15}$ They used a molecular weight of PMMA ( $\left.85 \mathrm{kDa}\right)$ similar to our high molecular weight $(92 \mathrm{kDa})$. However, since the temperature $\left(130^{\circ} \mathrm{C}\right)$ of the previous study was different from our diffusion temperature $\left(145^{\circ} \mathrm{C}\right)$, we applied Williams-Landel-Ferry (WLF) equation to calculate $D_{\text {bulk }}$ for $145^{\circ} \mathrm{C}^{16}$,

$$
\log \left(\frac{D\left(T_{0}\right)}{D\left(T_{1}\right)}\right)=\frac{-C_{1}\left(T-T_{0}\right)}{C_{2}+T-T_{0}}
$$

where, $C_{1}=11.9$, and $C_{2}=69 \mathrm{~K} \cdot{ }^{17} D_{\text {bulk }}$ is calculated to be $0.551 \times 10^{17} \mathrm{~cm}^{2} \mathrm{~s}^{-1}$ at $145^{\circ} \mathrm{C}$. This is similar to our diffusion coefficients of thick films. 
Table S1. Thickness of each layer consisting of PS/PMMA/dPMMA/PS from the NR results.

\begin{tabular}{|c|c|c|c|c|c|}
\hline \multicolumn{7}{|c|}{ Symmetry geometry sample } \\
\hline$d$ PMMA thick (Rg) & Bottom PS $(\AA)$ & $d$ PMMA $(\AA)$ & PMMA $(\AA)$ & Top PS $(\AA)$ & $\begin{array}{c}\text { PS/PMMA } \\
\text { Interface width } \\
(\AA)\end{array}$ \\
\hline $\mathbf{2 . 1}$ & 182 & 177 & 170 & 188 & 26 \\
\hline $\mathbf{4 . 4}$ & 198 & 368 & 319 & 196 & 26 \\
\hline $\mathbf{5 . 0}$ & 178 & 420 & 429 & 150 & 20 \\
\hline $\mathbf{6 . 5}$ & 184 & 544 & 484 & 187 & 27 \\
\hline $\mathbf{8 . 5}$ & 184 & 713 & 747 & 190 & 28 \\
\hline
\end{tabular}

\begin{tabular}{|c|c|c|c|c|c|}
\hline \multicolumn{7}{|c|}{ Asymmetry geometry sample } \\
\hline dPMMA thick (Rg) & Bottom PS $(\AA)$ & $d$ PMMA $(\AA)$ & PMMA $(\AA)$ & Top PS $(\AA)$ & $\begin{array}{c}\text { P.PMA } \\
\text { Interface width } \\
(\AA)\end{array}$ \\
\hline $\mathbf{1 . 2}$ & 191 & 98 & 1069 & 191 & 23 \\
\hline $\mathbf{2 . 4}$ & 180 & 205 & 984 & 180 & 29 \\
\hline $\mathbf{3 . 6}$ & 186 & 299 & 819 & 202 & 13 \\
\hline $\mathbf{4 . 9}$ & 202 & 413 & 772 & 193 & 24 \\
\hline $\mathbf{6 . 0}$ & 182 & 503 & 621 & 205 & 25 \\
\hline $\mathbf{7 . 0}$ & 198 & 587 & 548 & 199 & 25 \\
\hline
\end{tabular}


Table S2. Fitting parameter of the Neutron reflectivity measurement results with symmetric/asymmetric sample of PS/PMMA/dPMMA/PS.

\begin{tabular}{|c|c|c|c|c|c|c|c|c|c|c|c|c|c|c|}
\hline \multicolumn{15}{|c|}{ Symmetry geometry sample } \\
\hline \multirow{2}{*}{$\underset{(\min )}{\mathrm{t}}$} & \multicolumn{2}{|c|}{$2.1 \mathrm{Rg}$} & \multirow{2}{*}{$\underset{(\min )}{t}$} & \multicolumn{2}{|c|}{$4.4 \mathrm{Rg}$} & \multirow[b]{2}{*}{$\underset{(\min )}{\mathbf{t}}$} & \multicolumn{2}{|c|}{$5.0 \mathrm{Rg}$} & \multirow{2}{*}{$\underset{(\min )}{\mathrm{t}}$} & \multicolumn{2}{|c|}{$6.5 \mathrm{Rg}$} & \multirow{2}{*}{$\underset{(\min )}{\mathbf{t}}$} & \multicolumn{2}{|c|}{$8.5 \mathrm{Rg}$} \\
\hline & $\begin{array}{c}\sigma \sigma \\
(\AA)\end{array}$ & $\mathbf{X}^{2}$ & & $\begin{array}{c}\sigma \\
(\AA) \\
(\AA)\end{array}$ & $\mathbf{X}^{2}$ & & $\begin{array}{c}\sigma \\
(\AA)\end{array}$ & $\mathbf{X}^{2}$ & & $\begin{array}{c}\sigma \\
(\AA)\end{array}$ & $\mathbf{X}^{2}$ & & $\begin{array}{c}\sigma \\
(\AA)\end{array}$ & $\mathbf{X}^{2}$ \\
\hline 0 & 66.1 & 0.99 & 0 & 67.2 & 0.70 & 0 & 4 & 0 & 0 & 49.6 & 0.91 & 0 & 36.8 & 1.36 \\
\hline 40 & 85.6 & 1.18 & 42 & 93.0 & 0.97 & 42 & 23.4 & 70 & 60 & 53.2 & 1.71 & 70 & 43.5 & 1.19 \\
\hline 80 & 105 & 1.29 & 95 & 108 & 1.89 & 95 & 27.7 & 140 & 120 & 58.2 & 4.32 & 140 & 53.6 & 1.65 \\
\hline 120 & 125 & 1.12 & 140 & 131 & 2.4 & 140 & 30.6 & 280 & 180 & 66.8 & 4.01 & 280 & 68.0 & 1.51 \\
\hline \multirow[t]{8}{*}{160} & 135 & 0.71 & 185 & 145 & 0.90 & 185 & 33.8 & 350 & 240 & 66.8 & 4.01 & 350 & 73.9 & 1.81 \\
\hline & & & 230 & 164 & 0.93 & 230 & 37.2 & 420 & 300 & 74.1 & 3.54 & 420 & 84.0 & 0.91 \\
\hline & & & 275 & 186 & 0.90 & 275 & 41.5 & 490 & 360 & 79.0 & 3.4 & 490 & 90.7 & 0.87 \\
\hline & & & 320 & 207 & 1.08 & 320 & 44.7 & 560 & 420 & 87.5 & 2.56 & 560 & 94.1 & 0.89 \\
\hline & & & 365 & 191 & 0.78 & 365 & 47.6 & 2.98 & 480 & 91.4 & 2.83 & & & \\
\hline & & & 410 & 218 & 0.84 & 410 & 47.6 & 2.54 & 540 & 96.5 & 3.04 & & & \\
\hline & & & 455 & 222 & 0.70 & 455 & 47.9 & 2.40 & 600 & 102 & 3.20 & & & \\
\hline & & & 500 & 245 & 1.3 & & & & & & & & & \\
\hline
\end{tabular}

\begin{tabular}{|c|c|c|c|c|c|c|c|c|c|c|c|}
\hline \multicolumn{12}{|c|}{ Asymmetry geometry sample } \\
\hline \multirow{2}{*}{$t(\min )$} & \multicolumn{2}{|c|}{$1.2 \mathrm{Rg}$} & \multirow{2}{*}{$\mathbf{t}(\min )$} & \multicolumn{2}{|c|}{$2.4 \mathrm{Rg}$} & \multirow{2}{*}{$\mathbf{t}(\mathbf{m i n})$} & \multicolumn{2}{|c|}{$3.6 \mathrm{Rg}$} & \multirow{2}{*}{$\mathbf{t}(\min )$} & \multicolumn{2}{|c|}{$4.9 \mathrm{Rg}$} \\
\hline & $\sigma(\AA)$ & $\mathrm{X}^{2}$ & & $\sigma(\AA)$ & $\mathrm{X}^{2}$ & & $\sigma(\AA)$ & $\mathrm{X}^{2}$ & & $\sigma(\AA)$ & $\mathrm{X}^{2}$ \\
\hline 0 & 29.6 & 3.50 & 0 & 12.0 & 3.46 & 0 & 15.3 & 3.27 & 0 & 4.02 & 2.60 \\
\hline 37 & 41.7 & 3.42 & 37 & 20.8 & 1.56 & 37 & 19.6 & 1.80 & 37 & 28.1 & 2.99 \\
\hline 74 & 53.4 & 3.79 & 74 & 24.7 & 1.42 & 74 & 21.4 & 1.77 & 74 & 39.0 & 2.94 \\
\hline 111 & 73.4 & 3.46 & 111 & 27.3 & 1.42 & 111 & 22.5 & 1.72 & 111 & 48.0 & 3.16 \\
\hline \multirow[t]{12}{*}{148} & 80.2 & 4.44 & 148 & 29.4 & 1.74 & 148 & 23.3 & 1.77 & 148 & 54.5 & 3.17 \\
\hline & & & 185 & 32.2 & 1.98 & 185 & 25.1 & 1.72 & 185 & 61.5 & 2.95 \\
\hline & & & 222 & 34.7 & 1.88 & 222 & 26.0 & 1.80 & 222 & 67.5 & 2.83 \\
\hline & & & 259 & 36.7 & 1.83 & 259 & 26.6 & 1.64 & 259 & 72.0 & 2.83 \\
\hline & & & 296 & 39.5 & 2.18 & 296 & 28.0 & 2.01 & 296 & 76.8 & 2.73 \\
\hline & & & 333 & 41.5 & 2.16 & 333 & 28.6 & 2.10 & 333 & 78.8 & 2.49 \\
\hline & & & 370 & 43.6 & 2.25 & 370 & 29.4 & 1.86 & 370 & 82.8 & 2.46 \\
\hline & & & & & & 407 & 30.1 & 2.09 & & & \\
\hline & & & & & & 444 & 30.7 & 2.09 & & & \\
\hline & & & & & & 481 & 32.5 & 2.08 & & & \\
\hline & & & & & & 518 & 33.2 & 2.23 & & & \\
\hline & & & & & & 555 & 33.7 & 2.21 & & & \\
\hline
\end{tabular}

\begin{tabular}{|c|c|c|c|c|c|}
\hline \multirow{2}{*}{$\begin{array}{c}\mathbf{t} \\
(\mathrm{min})\end{array}$} & \multicolumn{2}{|c|}{$6.0 \mathrm{Rg}$} & \multirow{2}{*}{$\underset{(\mathbf{m i n})}{\mathbf{t}}$} & \multicolumn{2}{|c|}{$7.0 \mathrm{Rg}$} \\
\hline & $\begin{array}{c}\sigma \\
(\AA)\end{array}$ & $\mathbf{X}^{2}$ & & $\begin{array}{c}\sigma \\
(\AA)\end{array}$ & $X^{2}$ \\
\hline 0 & 6.0 & 4.08 & 0 & 24.0 & 4.33 \\
\hline - & - & - & 37 & 37.1 & 3.59 \\
\hline 74 & 41.5 & 4.48 & 74 & 47.2 & 3.47 \\
\hline 111 & 59.4 & 4.13 & 111 & 56.1 & 3.45 \\
\hline 148 & 68.2 & 4.16 & 148 & 64.2 & 3.43 \\
\hline 185 & 75.0 & 3.85 & 185 & 70.2 & 3.43 \\
\hline 222 & 81.7 & 3.85 & 222 & 76.1 & 3.27 \\
\hline 259 & 81.7 & 3.81 & 259 & 82.5 & 3.27 \\
\hline 296 & 87.0 & 3.82 & 296 & 86.5 & 3.29 \\
\hline 333 & 93.3 & 3.60 & 333 & 92.0 & 3.24 \\
\hline 370 & 98.6 & 3.60 & 370 & 92.9 & 3.54 \\
\hline 407 & 100 & 3.60 & & & \\
\hline
\end{tabular}




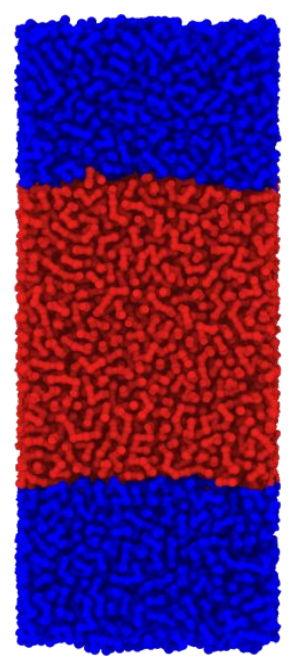

Figure S1. Representative snapshot of our simulations. Blue layers represent type A polymers that mimic PS and the red center layer represent type B polymers that mimic PMMA. 


\section{REFERENCE}

1. Ennis, D.; Betz, H.; Ade, H., Direct spincasting of polystyrene thin films onto poly (methyl methacrylate). J. Polym. Sci., Part B: Polym. Phys. 2006, 44 (22), 3234-3244.

2. Yoshimoto, K.; Jain, T. S.; Van Workum, K.; Nealey, P. F.; de Pablo, J. J., Mechanical heterogeneities in model polymer glasses at small length scales. Phys. Rev. Lett. 2004, 93 (17), 175501.

3. Riggleman, R. A.; Yoshimoto, K.; Douglas, J. F.; de Pablo, J. J., Influence of confinement on the fragility of antiplasticized and pure polymer films. Phys. Rev. Lett. 2006, 97 (4), 045502.

4. Riggleman, R. A.; Douglas, J. F.; de Pablo, J. J., Tuning polymer melt fragility with antiplasticizer additives. J. Chem. Phys. 2007, 126 (23), 234903.

5. Riggleman, R. A.; Douglas, J. F.; de Pablo, J. J., Antiplasticization and the elastic properties of glass-forming polymer liquids. Soft Matter 2010, 6 (2), 292-304.

6. Im, H.; Oh, Y.; Cho, H. W.; Kim, J.; Paeng, K.; Sung, B. J., The spatial arrangement of a single nanoparticle in a thin polymer film and its effect on the nanoparticle diffusion. Soft matter 2017, 13 (35), 5897-5904.

7. Karatrantos, A.; Clarke, N.; Composto, R. J.; Winey, K. I., Topological entanglement length in polymer melts and nanocomposites by a DPD polymer model. Soft Matter 2013, 9 (14), 3877-3884.

8. Weeks, J. D.; Chandler, D.; Andersen, H. C., Role of repulsive forces in determining the equilibrium structure of simple liquids. J. Chem. Phys. 1971, 54 (12), 5237-5247.

9. Plimpton, S., Fast Parallel Algorithms for Short-Range Molecular Dynamics. J. Comp. Phys. 1995, 117 (1), 1-19.

10. Zhang, W.; Douglas, J. F.; Starr, F. W., Why we need to look beyond the glass transition temperature to characterize the dynamics of thin supported polymer films. Proc. Natl. Acad. Sci. U. S. A. 2018, 115 (22), 5641-5646.

11. Russell, T., X-ray and neutron reflectivity for the investigation of polymers. Mater. Sci. Rep. 1990, 5 (4), 171-271.

12. Marquardt, D. W., An algorithm for least-squares estimation of nonlinear parameters. $Q$. Appl. Math. 1963, 11 (2), 431-441.

13. Levenberg, K., A method for the solution of certain non-linear problems in least squares. SIAM J. Appl. Math. 1944, 2 (2), 164-168.

14. Rubinstein, M.; Colby, R., Polymer Physics, Oxford University Press. New York 2003.

15. Kunz, K.; Stamm, M., Initial Stages of Interdiffusion of PMMA across an Interface. Macromolecules 1996, 29 (7), 2548-2554.

16. Ferry, J. D., Viscoelastic properties of polymers. John Wiley \& Sons: 1980.

17. Colby, R. H., Breakdown of time-temperature superposition in miscible polymer blends. Polymer 1989, 30 (7), 1275-1278. 